\title{
ALL-OPTICAL NETWORK DESIGN WITH EVEN AND ODD NODES
}

\author{
Pritam Keshari Sahoo $^{1}$, Madhuli Madhusmita Das ${ }^{2}$ \\ ${ }^{1}$ Lecturer, Electronics and Tele-comm. Engineering Dept., Seemanta Engg College, Odisha, India \\ ${ }^{2}$ Asst. professor, Electronics and Tele-comm. Engineering Dept., Orissa Engineering College, Odisha ,India
}

\begin{abstract}
We have studied the effects of OLTs and OADMs in WDM optical networks. All optical networks have proved to be cost efficient and power saving in comparison to $O-E-O$ networks. Cost of a network can further be reduced by minimizing the number of IP router ports and the number of wavelengths required. It has been already studied the number of IP router ports required per node and number of wavelengths required to carry a fixed amount of traffic, considering the network containing even and odd number of routing nodes. And finally the result has been compared with other architectures like point to point WDM and hub networks, finally all-optical networks proved to be most cost efficient in saving number of wavelength requirements and IP router port requirements. In this paper we have compared all-optical network with itself, by taking even and odd number of nodes. That is we have compared all-optical network containing even number of nodes with the same all-optical network containing odd number of nodes. The result what we obtained is to honor same amount of traffic " $t$ ", all-optical network containing odd number of nodes require lesser number of wavelengths than its previous even number of nodes. We have here varied the number of nodes keeping the amount of traffic fixed assuming static routing for simplicity of our work. Finally we observed the percentage of change in wavelength requirements decreases on increasing number of nodes. That is for a large network number of wavelength requirements are large for even number of nodes than odd number of nodes. But this difference is little more for a small network size.
\end{abstract}

Keywords: Wavelength Division Multiplexing (WDM), PPWDM (Point to point WDM), Light paths, traffic, alloptical, Erlang(E).

\section{INTRODUCTION}

Wavelength Division Multiplexing (WDM) provides capability of building a large network of high capacity. Fig1 shows an all-optical network employing wavelength routing, consisting of optical router nodes interconnected by optical links. Each link is a bidirectional consists of two unidirectional links. But two light paths can't posses same wavelength within a single link. If there are $\Lambda$ wavelengths on each link, hence $\Lambda$ independent switches required per each node for each light path. Each switch is having $\Delta$ input and $\Delta$ output ports. In an all-optical network there is no necessity of optical to electrical conversion and vice-versa. In a WDM network these nodes are act like a source or sink of light paths. More theoretical studies of these networks appear in [3], [4].

In the network as shown in fig-1, connections arrive and terminate at random. And each connection requires a path from source node to destination node with a specific wavelength. The assigned wavelength must remain same throughout the path. A connection request from node $\mathrm{A}$ to $\mathrm{C}$ is assigned wavelength $\lambda_{1}$ and an another request from $C$ to $\mathrm{E}$, which uses a different path is assigned same wavelength $\lambda_{1}$, but the request from $\mathrm{B}$ to $\mathrm{D}$, that interferes the path from A to $C$ and from $C$ to $E$ is assigned another wavelength $\lambda_{2}$. Several routing and wavelength assignments are considered in [5]-[7].

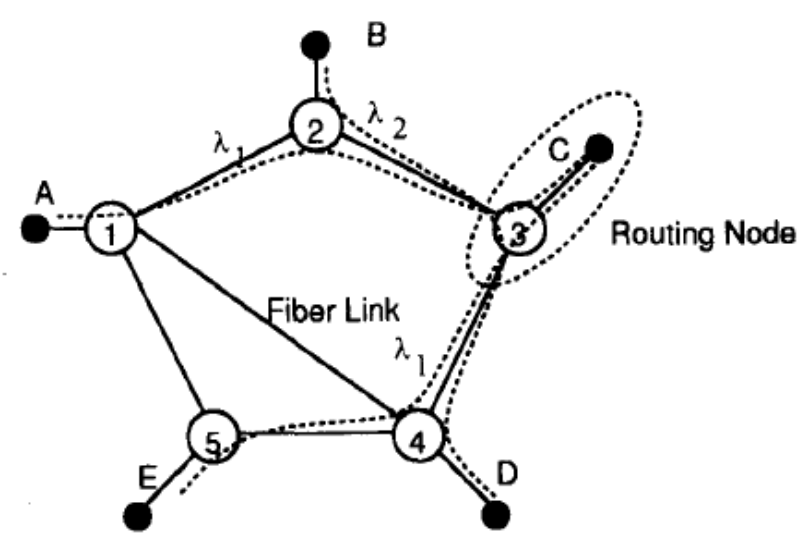

Fig-1: A Simple WDM network

Related works to determine the number of wavelengths and IP router ports required to support a specific traffic has been analyzed [8].

We'll take a network where traffic must be routed from source to destination node on a single light path. No intermediate nodes are required to add or drop any wavelength electrically (O-E-O). Each lightpath follows a route in the network and is assigned a wavelength along each link in the path [1].

We consider static uniform traffic. It has an integer parameter $g$ and has an exactly $g$ traffic streams between every pair of nodes. The traffic distribution matrix will be represented by a matrix $T=[T(i, j)] . T(i, j)$ is the number of 
lightpaths of traffic between node $\mathrm{i}$ and $\mathrm{j}$. Where $\mathrm{c} \cdot \mathrm{T}(\mathrm{i}, \mathrm{j})$ equals the number of traffic streams between node $i$ and $j$. Thus $g=c \cdot T(i, j)$. Or $T(i, j)=g / c$ if $i \neq j$ and $T(i, j)=0$ if $i=j$.

In this paper we have presented an analytical method to calculate number of wavelengths required for an all-optical network. In section 2, we present an all-optical WDM network and calculated the wavelength requirements in case of even number of nodes in section 2.1 and odd number of nodes in section 2.2. The results are compared between themselves in section 3 and final conclusion is presented in section 4 .

\section{ALL-OPTICAL ARCHITECTURE}

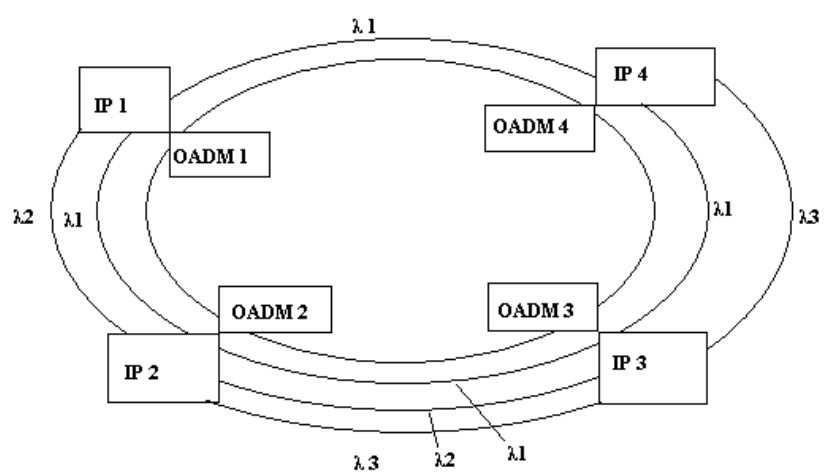

Fig-2: An all-optical network for case $\mathrm{t}=3$.

The final architecture is the all-optical design as shown in figure. Here data is transmitted on a single light path between its source and destination, never sent through an intermediate router. In this case we must set up $\lceil\mathrm{t} /(\mathrm{N}-1)\rceil$ light paths between each pair of nodes to handle $t /(\mathrm{N}-1)$ units of traffic between each node pair. The number of router ports per node is

$$
\mathrm{Q}=(\mathrm{N}-1)\lceil\mathrm{t} / \mathrm{N}-1\rceil
$$

The number of wavelengths required is obtained from following analysis.

\subsection{Consider $\mathbf{N}=$ even. [8]}

Start with 2 nodes on the ring. The light path needs one wavelength.

Let $\mathbf{k}$ is the number of nodes present currently in the ring.

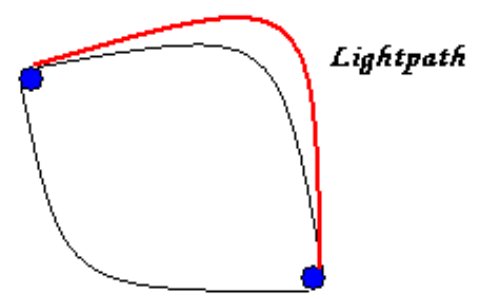

Fig-3: all-optical network for $\mathrm{k}=2$
Add two nodes to the ring till $\mathrm{k}<=\mathrm{N}-2$. And add two new nodes diametrically which divide the ring symmetrically and each contains $\mathrm{k} / 2$ number of old nodes.

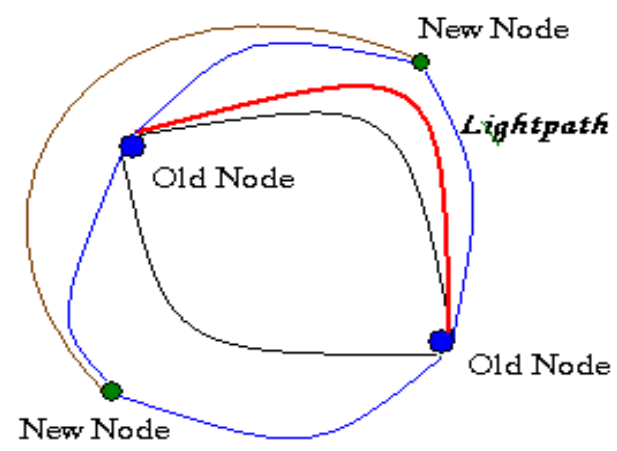

Fig-4: all-optical network for $\mathrm{k}=2$ and $\mathrm{N}=4$.

In one half each node creates a light path to each of the new nodes. This requires one wavelength by reusing the wavelength. Hence a total of $\mathrm{k} / 2$ wavelengths are required. In the other half same $\mathrm{k} / 2$ wavelengths are required. And two new nodes require one wavelength to set up a light path between them. So total $(\mathrm{k} / 2)+1$ new wavelengths are required, as shown in figure below for $\mathrm{k}=4$ and again two new nodes are added, so that $\mathrm{N}=6$.

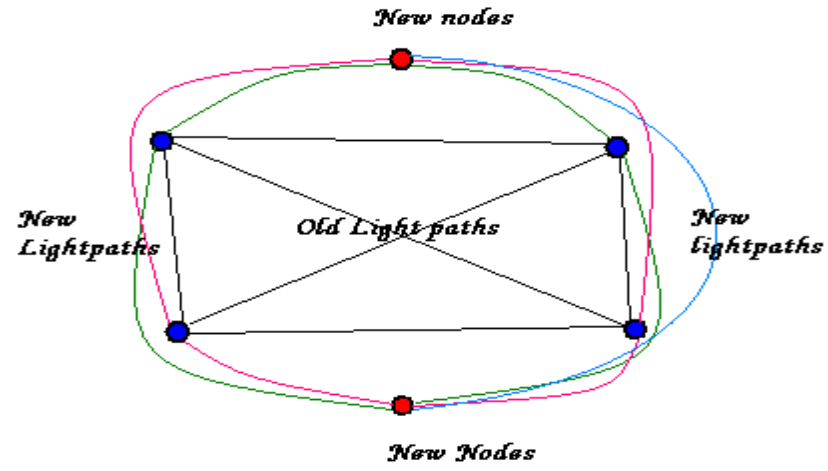

Fig-5: all-optical network for $\mathrm{k}=4$ and $\mathrm{N}=6$.

As we have considered $\mathrm{N}=$ even, for

$\mathrm{k}=0,2,4,6$.

Number of wavelengths required for wavelength assignment is

$\mathrm{W}=1+2+3+\ldots \ldots \ldots \ldots \ldots \ldots \ldots+(\mathrm{N} / 2)=\frac{N^{2}}{8}+\frac{N}{4}$

For some fixed traffic $\left(\frac{t}{N-1}\right)$ between two nodes

$$
\mathbf{W}=\left\lceil\frac{t}{N-1}\right\rceil\left(\frac{N^{2}}{8}+\frac{N}{4}\right)
$$




\subsection{Consider $\mathrm{N}=\mathbf{O d d}$.}

Start for $\mathrm{N}=3$ and add 2 nodes each time. As shown in figure for 3 nodes only 1 wavelength is required for wavelength assignment. Now on adding two more nodes there is requirement of 2 more wavelengths. For doing $\mathrm{N}=5,7$ and so on the net increase in Wavelengths is $\frac{k+1}{2}$.

And $\mathrm{k}=1,3,5$,

$\mathrm{W}=1+2+3+$ $+\left(\frac{N-2+1}{2}\right)$

$=\frac{1}{2}\left(\frac{N-1}{2}\right)\left(\frac{N+1}{2}\right)$

$\mathrm{W}=\frac{1}{8}\left(N^{2}-1\right)$

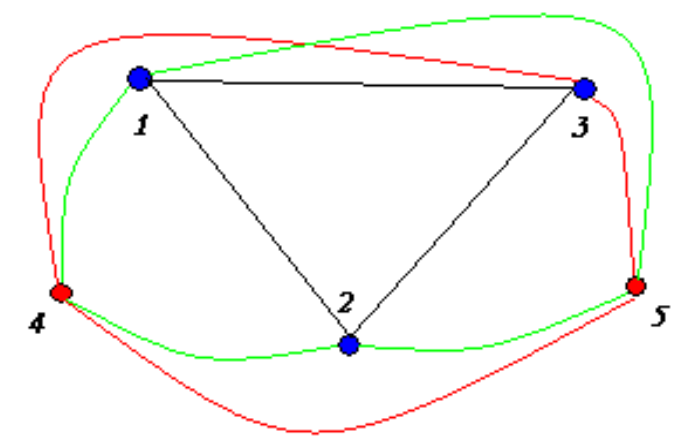

Fig-6: all-optical network for $\mathrm{k}=3$ and $\mathrm{N}=5$.

For some fixed traffic $\left(\frac{t}{\mathbb{W}-1}\right)$ between two nodes

$$
\mathrm{W}=\frac{1}{8}\left\lceil\frac{t}{N-1}\right\rceil\left(N^{2}-1\right)
$$

\section{PERFORMANCE ANALYSIS}

The results of section 2.1 and 2.2 from [8] are the number of wavelengths required to honor a fixed amount of traffic " $t$ ".

This result of all-optical network is most cost effective among other network architectures like PPWDM ring, HUB ring etc.

In order to set up an all-optical network for a service provider, number of routing nodes has to be defined by the intermediated destinations as well as the source and final destination. Hence we have here studied the performance of the network with even and odd number of nodes, by comparing eq-(1) and eq-(2) based on least number of wavelength requirements.

Assume the total traffic/load offered to the network $\mathrm{t}=5 \mathrm{E}$.
Table-1: Node Vs Wavelength table

\begin{tabular}{|c|c|c|c|}
\hline $\mathbf{N}=$ Even & Wavelengths & $\mathbf{N}=$ Odd & Wavelengths \\
\hline 2 & 5 & 3 & 3 \\
\hline 4 & 6 & 5 & 6 \\
\hline 6 & 6 & 7 & 6 \\
\hline 8 & 10 & 9 & 10 \\
\hline 10 & 15 & 11 & 15 \\
\hline 12 & 21 & 13 & 21 \\
\hline 14 & 28 & 15 & 28 \\
\hline 16 & 36 & 17 & 36 \\
\hline 18 & 45 & 19 & 45 \\
\hline 20 & 55 & 21 & 55 \\
\hline 22 & 66 & 23 & 66 \\
\hline
\end{tabular}

We observed from the above table that, there is no requirement of additional wavelengths when a service provider deploys one more node from earlier existing even nodes. Where as a significant additional requirement of wavelengths occur when the service provider deploys one more node from its existing odd nodes. Each time when a new node is added to existing odd nodes, the rate of increase in wavelength requirement is a gradual fall.

The plots of rate of increase in wavelengths for even and odd number of nodes are drawn. From the plots of Fig: 7 and Fig: 8, rate of increase in wavelength remains same in both cases. That is there is no additional cost requirement for an additional node from even to odd except equipmental cost.

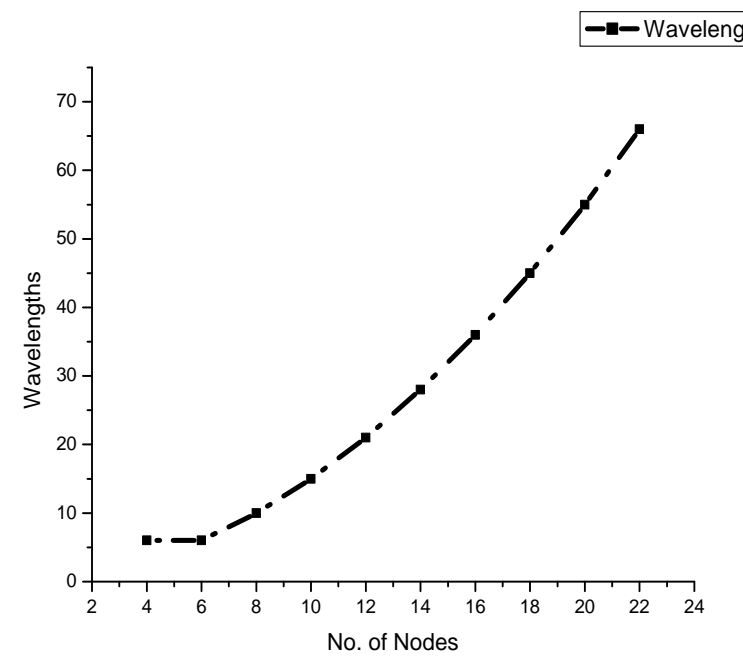

Fig-7: Wavelength Vs Nodes for $\mathrm{N}=$ even 


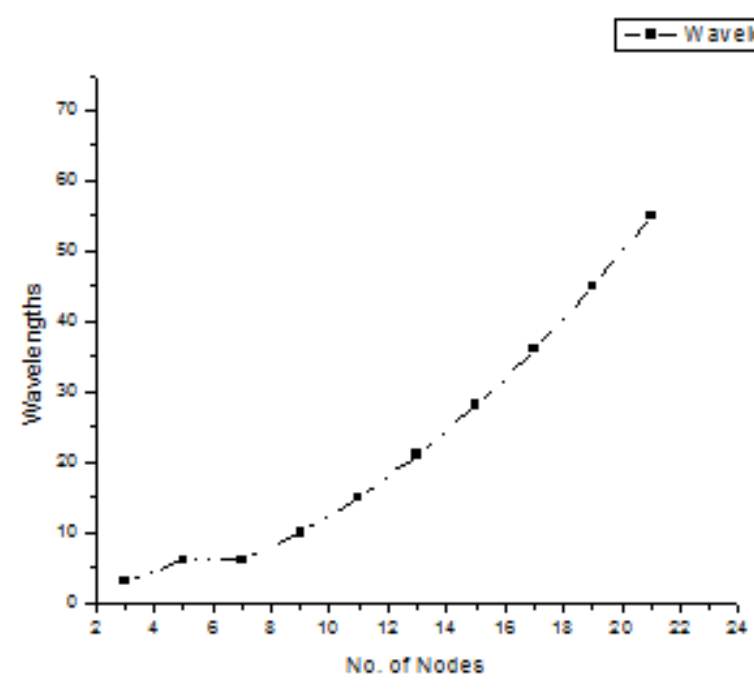

Fig-8: Wavelength Vs Nodes for $\mathrm{N}=\mathrm{Odd}$

\section{CONCLUSION}

Even though all-optical network has proved to be most cost effective network architecture from power consumption point of view as well as lesser wavelength requirement, an all-optical network with odd number of nodes can create one more source/destination terminal than its previous even node utilizing the same wavelengths and to honor the same traffic load.

\section{REFERENCES}

[1]. R. Ramaswami and G. H. Sasaki, "Multiwavelength optical networks with limited wavelength conversion," IEEE/ACM Trans. Networking, vol. 6, pp. 744-754, Dec. 1998.

[2]. R. Ramaswami and K. N. Sivarajan. "Routing and wavelength assignment in all-optical networks," IEEE/ACM Trans. Networking, Vol. 3, no. 5, Oct 1995.

[3]. I. chlamtac, a. gam, and g. karmi, "Purely optical networks for terabit communication," in proc. IEEE infocom'89, 1989, pp. 887-896.

[4] A. Aggarwal, A. Bar-Noy, D. Coppersmith, R. Ramaswami, B. Schieber, and M. Sudan, "Efficient routing and scheduling algorithms for optical networks," in Proc. 5th Annu. ACM-SIAM Symp. Discrete Algorithms,Jan. 1994, pp 412423.

[5]. K. Bala, T. E. Stem, and K. Bala,"Algorithms for routing in a linear lightwave network," in Proc. INFOCOM'91, 1991, pp. 1-9.

[6]. K. Bala, "Routing in linear lightwave Networks," Ph.D. dissertation, Columbia Univ., 1993, CTR Tech. Rep. 32393-02.

[7]. I. Chlamtac, A. Ganz, and G. Karmi, "Lightpath communications: An approach to high-bandwidth optical WAN'S,' IEEE Trans. Commun. vol. 40, pp. 1171-1182, July 1992.

[8]. O. Gerstel, R. Ramaswami and G. H. Sasaki,"Costeffective traffic grooming in WDM ring," IEEE/ACM Trans on networking, Vol. 8, No. 5, Oct. 2000.

\section{BIOGRAPHIES}

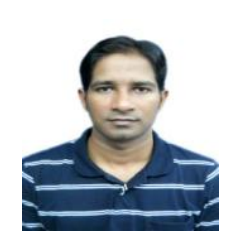

Pritam Keshari Sahoo has received his M.Tech degree from IIT, Kharagpur. He has seven years of teaching experience in Seemanta Engg. College, Odisha as Lecturer. His research interests are Optical Communication and Networking, Wireless Communication. He is the lifetime member of ISTE.

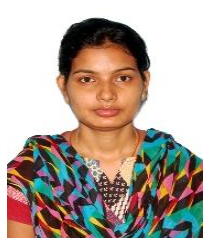

Madhuli Madhusmita Das has received her M.Tech degree from IIT, Kharagpur. She has six years of teaching experience in Orissa Engg. College, Odisha as Asst. Professor. Her research interests are Optical Communication and Networking. 